\title{
Large process-dependent variations in band alignment and interface band gaps of Cu2ZnSnS4/CdS solar cells
}

\author{
Crovetto, Andrea; Cazzaniga, Andrea; Ettlinger, Rebecca Bolt; Schou, Jørgen; Hansen, Ole
}

Published in:

Solar Energy Materials and Solar Cells

Link to article, DOI:

10.1016/j.solmat.2018.08.005

Publication date:

2018

Document Version

Early version, also known as pre-print

Link back to DTU Orbit

Citation (APA):

Crovetto, A., Cazzaniga, A., Ettlinger, R. B., Schou, J., \& Hansen, O. (2018). Large process-dependent variations in band alignment and interface band gaps of $\mathrm{Cu}_{2} \mathrm{ZnSnS} / \mathrm{CdS}$ solar cells. Solar Energy Materials and Solar Cells, 187, 233-240. https://doi.org/10.1016/j.solnfat.2018.08.005

\section{General rights}

Copyright and moral rights for the publications made accessible in the public portal are retained by the authors and/or other copyright owners and it is a condition of accessing publications that users recognise and abide by the legal requirements associated with these rights.

- Users may download and print one copy of any publication from the public portal for the purpose of private study or research.

- You may not further distribute the material or use it for any profit-making activity or commercial gain

- You may freely distribute the URL identifying the publication in the public portal 


\title{
Large process-dependent variations in band alignment and interface band gaps of $\mathrm{Cu}_{2} \mathrm{ZnSnS}_{4} / \mathrm{CdS}$ solar cells
}

\author{
Andrea Crovetto $^{\mathrm{a}, \mathrm{b}, *}$, Andrea Cazzaniga ${ }^{\mathrm{c}}$, Rebecca Bolt Ettlinger ${ }^{\mathrm{c}}, \mathrm{J} ø r g e n \mathrm{Schou}^{\mathrm{c}}$, Ole Hansen ${ }^{\mathrm{a}}$ \\ ${ }^{a}$ DTU Nanotech, Technical University of Denmark, DK-2800 Kgs. Lyngby, Denmark \\ ${ }^{b}$ SurfCat, Department of Physics, Technical University of Denmark, DK-2800 Kgs. Lyngby, Denmark \\ ${ }^{c}$ DTU Fotonik, Technical University of Denmark, DK-4000 Roskilde, Denmark
}

\begin{abstract}
Electron-hole recombination at the $\mathrm{Cu}_{2} \mathrm{ZnSnS}_{4} / \mathrm{CdS}$ interface is believed to play a major role in limiting the efficiency of $\mathrm{Cu}_{2} \mathrm{ZnSnS}_{4}$ solar cells. In this work, we experimentally determine detailed $\mathrm{Cu}_{2} \mathrm{ZnSnS}_{4} / \mathrm{CdS}$ interface band diagrams as a function of process conditions, and correlate them to chemical processes occurring during interface formation and subsequent post-annealing. The newly devised experimental method involves a combination of photoemission spectroscopy and spectroscopic ellipsometry. Our measurements reveal that, under most process conditions, the band gaps of both $\mathrm{Cu}_{2} \mathrm{ZnSnS}_{4}$ and $\mathrm{CdS}$ decrease by several hundred meV near the interface. Furthermore, interface band bending and conduction band offsets are highly process-dependent and roughly correlated to the amount of chemical interdiffusion. The interface electronic properties are found to be unfavorable under all process conditions studied in this work, either due to a cliff-like conduction band offset, or to substantial band gap narrowing in $\mathrm{Cu}_{2} \mathrm{ZnSnS}_{4}$, or to both effects. According to the present study, the least harmful process conditions for the interface electronic properties are a low CdS deposition temperature without post-annealing. Even in such a case, a minimum open circuit voltage loss of $230 \mathrm{mV}$ is expected due to interface- or near-interface recombination.
\end{abstract}

Keywords: CZTS, kesterite, interface, ellipsometry, photoemission spectroscopy, band alignment

\section{Introduction}

The $\mathrm{Cu}_{2} \mathrm{ZnSnS}_{4} / \mathrm{CdS}$ interface appears to be of crucial importance for the performance of $\mathrm{Cu}_{2} \mathrm{ZnSnS}_{4}$ (CZTS) solar cells. In fact, the open circuit voltage extrapolation to $0 \mathrm{~K}$ temperature, as reported by different research groups on different state-of-the-art CZTS/CdS solar cells, is consistently lower than the bulk band gap of CZTS, even when recombination from band tails in CZTS is taken into account $[1,2,3]$. The reason for this experimental finding is still debated and can be attributed, for example, to a clifflike conduction band alignment (CBO) between CZTS and CdS [4], to band gap narrowing of ideal CZTS surfaces [5], and to interdiffusion-driven band gap narrowing of CZTS near the interface with $\mathrm{CdS}[6,7]$. Even though problems with band alignment and band gap narrowing could also occur at the back contact interface $\left(\mathrm{MoS}_{2} / \mathrm{CZTS}\right)$, recent results point to a low work function of the back contact as the main issue [8].

To further complicate the issue, the properties of the CZTS/CdS interface could in principle be influenced by growth process conditions [7]. However, processdependent interface properties have only been studied for special cases, such as intentional $\mathrm{Zn}$ alloying in the $\mathrm{CdS}$

*Corresponding author, email: ancro@fysik.dtu.dk, Fysikvej, building 312, DK-2800 Kgs. Lyngby, Denmark, Tel.: +45 45253191 bulk [9], intentional Cd alloying in the CZTS bulk [3], and KCN etching of CZTS before CdS deposition [10]. One possible reason for the lack of systematic studies is the difficulty of determining the band gaps of the two materials near the heterointerface after interface formation. In fact, typical band alignment measurements based on direct photoemission spectroscopy can determine valence band offsets directly, but they usually rely on optical measurements of the two separate, bulk materials to determine their bulk band gaps [9, 3]. They assume therefore that the bulk band gaps of the two materials are equal to their band gaps in the interface region after interface formation. Even if complementary inverse photoemission spectroscopy measurements are performed to determine the surface band gaps of the two separate materials [10], the real interface band gaps may still be different from the surface band gaps due to interface-related phenomena such as interdiffusion, epitaxial growth, and early-stage growth mechanisms [7]. A post-annealing treatment of the asdeposited interfaces may further enhance the differences in electronic properties between the bulk materials and their near-interface region [6].

In order to characterize interface band gaps after interface preparation, we perform complementary spectroscopic ellipsometry measurements on CTZS/CdS film stacks. The ellipsometry spectra are sensitive to changes in the optical functions of the materials perpendicularly to the sub- 
strate plane; thus a band gap gradient in the out-of-plane direction can be detected by fitting the ellipsometry spectra to an appropriate multi-layer optical model [11, 12]. Complete interface band diagrams can then be drawn by combining the measured interface band gaps with the valence band offsets measured by x-ray photoemission spectroscopy. The goal of this paper is to determine those interface band diagrams as a function of process conditions, and correlate them to interfacial chemical processes.

\section{Experimental details}

CZTS thin films (about $100 \mathrm{~nm}$ thick) were deposited on a soda lime glass substrate by pulsed laser deposition from a single stoichiometric CZTS target, tuning the laser fluence to obtain the desired $\mathrm{Cu}$-poor, Zn-rich composition as described previously [13]. Deposition of the typical Mo back contact was omitted in order to limit the number of free parameters necessary for fitting ellipsometry spectra [11]. The CZTS films are thinner than in typical solar cells in order to keep their roughness low. This precaution is important because high surface roughness can depolarize the incident beam used in the ellipsometry measurement and render subsequent analysis impossible $[14,15,11]$. With such thin films, there is a risk that interdiffusion from CdS into CZTS might occur over the full absorber thickness. However, it will be shown later in this paper that the depth of elemental interdiffusion into CZTS is only tens of $\mathrm{nm}$, in agreement with previous studies [7]. The possibly higher Na concentration in $100 \mathrm{~nm}-$ thick CZTS compared to thicker absorbers deposited on Mo-coated glass is not expected to significantly alter the optoelectronic properties, because the effects of $\mathrm{Na}$ incorporation into CZTS depend on Na concentration only below a certain concentration threshold (about $10^{19} \mathrm{~cm}^{-3}$ ), which has to be achieved in order to obtain efficient solar cells [16]. The as-deposited CZTS films were postannealed in a sulfur-containing atmosphere at $550^{\circ} \mathrm{C}$ for $10 \mathrm{~min}$. Additional process details and film characterization are available elsewhere [13]. CdS thin films (20-40nm) were deposited on CZTS by chemical bath deposition at $55^{\circ} \mathrm{C}, 75^{\circ} \mathrm{C}$, and $95^{\circ} \mathrm{C}$ on nominally identical glass/CZTS substrates. The solvent for $\mathrm{CdS}$ deposition was milli-Q water, the $\mathrm{Cd}$ source was $1.5 \mathrm{mM} \mathrm{CdSO}_{4}$, and the sulfur source was $70 \mathrm{mM}$ thiourea. To prevent homogeneous $\mathrm{Cd}(\mathrm{OH})_{2}$ precipitation, an ammonia solution was added to the bath as a complexing agent. $1.7 \mathrm{M}, 2.0 \mathrm{M}$ and $2.4 \mathrm{M}$ ammonia was used at 55,75 , and $95^{\circ} \mathrm{C}$ deposition temperature respectively, in order to account for the increasing $\mathrm{OH}^{-}$concentration with increasing temperature and thus keep deposition rate roughly constant with temperature [17]. If the ammonia content is not adjusted at the different deposition temperatures, the resulting higher rate of homogeneous precipitation at higher temperature may have a stronger influence on the interfacae properties than the increase in temperature itself [17]. In order to avoid temperature transients in the deposition, thiourea

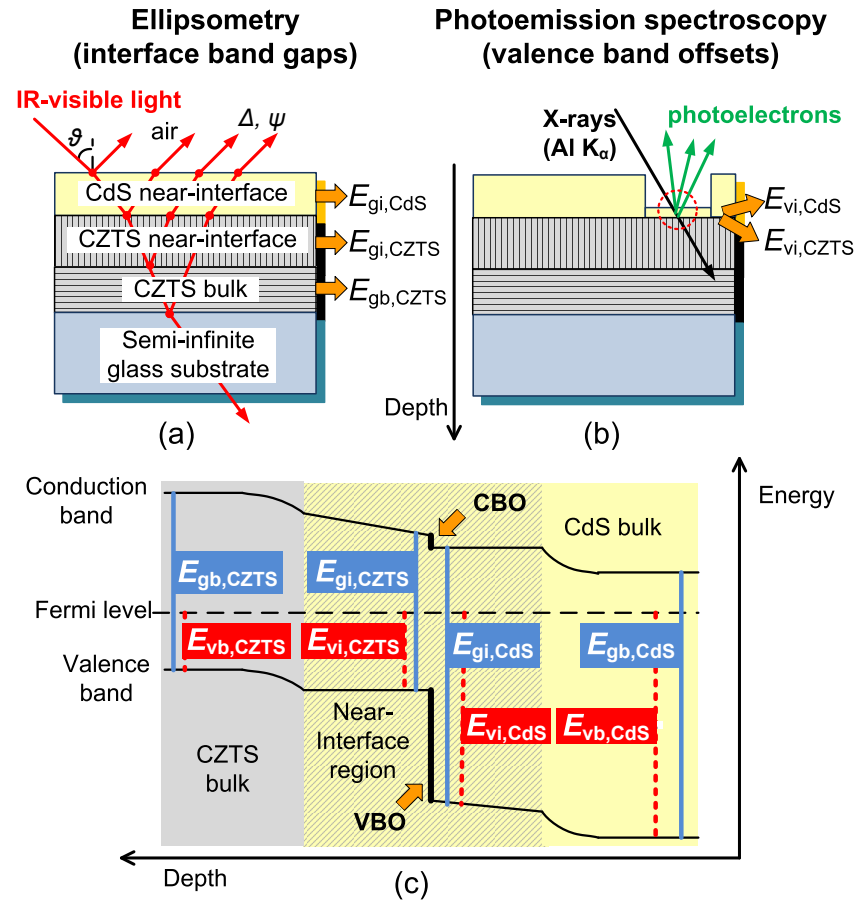

Figure 1: (a): Scheme of the optical model employed to extract band gaps by fitting ellipsometry spectra $\Psi(E)$ and $\Delta(E)$. The CZTS layer is split into sub-layers of equal thickness with independent band gaps. (b): Scheme of the XPS measurement employed to determine the valence band energy with respect to the Fermi level at different depths. The depth resolution is achieved by alternating an ion beam etching step with a photoemission measurement step. An additional output of the measurement is the depth-dependent chemical composition of $\mathrm{Cd}, \mathrm{Cu}, \mathrm{Zn}, \mathrm{Sn}, \mathrm{S}$. (c): Key to interpret the interface band diagrams plotted in this study. The quantities colored red (blue) are obtained by the XPS (ellipsometry) measurement. More details about the experimental method are available elsewhere [12].

was only added after the solution reached the desired temperature. A deposition time around 5 min yielded CdS films of $31 \mathrm{~nm}$ average thickness, with $8 \mathrm{~nm}$ standard deviation across samples. After CdS deposition, each of the three samples was cut in two halves, and one half of each sample was further annealed at $300^{\circ} \mathrm{C}$ for $20 \mathrm{~min}$ in $\mathrm{Ar}$, as reported previously [6]. This yielded a total of six samples for interface electronic characterization. Note that the CZTS bulk in one of the six samples is more Cu-rich than what was intended. This could be due to the reproducibility issues of PLD discussed in [13] or to the dependence of film composition on the exact position of the substrate in the PLD chamber. The three samples that were not postannealed will be denoted "as-deposited" in the following.

Variable-angle spectroscopic ellipsometry measurements were performed to obtain the CZTS band gap in the bulk $E_{\text {gb,CZTS }}$, and the CZTS (CdS) band gaps near the interface $E_{\text {gi,CZTS }}\left(E_{\text {gi,CdS }}\right)$ as shown in Fig. $1(\mathrm{a})$. The measurement was performed with a rotating compensator spectroscopic ellipsometer (M-2000, J.A. Woollam Co.) at six different angles $\theta\left(45-70^{\circ}\right)$, with a spot size of about $200 \mu \mathrm{m} \times 300 \mu \mathrm{m}$. Incident light of known polarization 


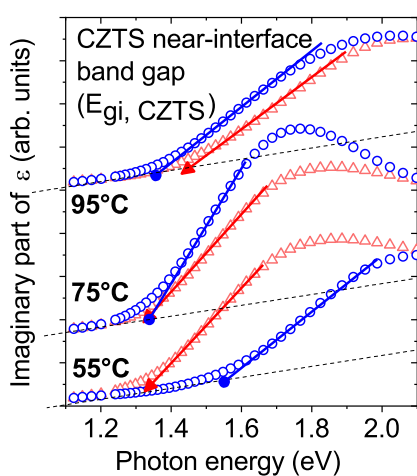

(a)

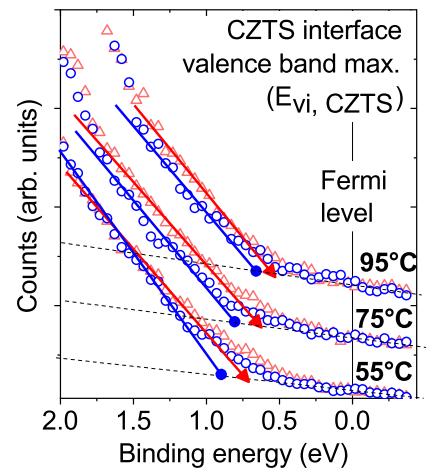

(c)

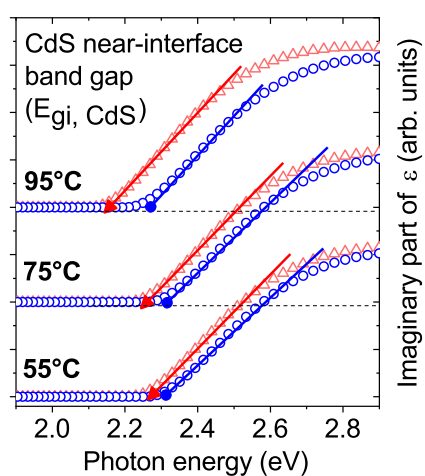

(b)

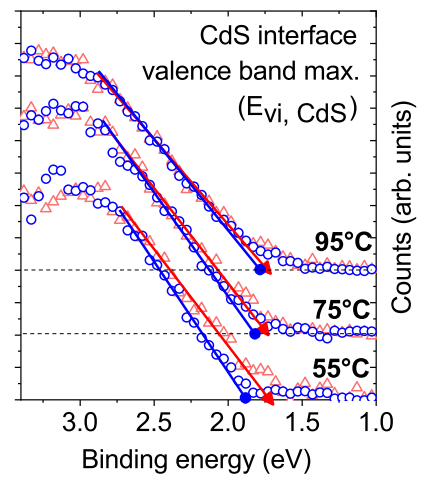

(d)
Figure 2: $(\mathrm{a}, \mathrm{b})$ : The fitted imaginary part of the dielectric function of the CZTS near-interface layer (a) and of the CdS near-interface layer (b), where we refer to the layer structure of Fig. 1(a). The nearinterface band gaps of the two materials $\left(E_{\mathrm{gi}, \mathrm{CZTS}}\right.$ and $E_{\mathrm{gi}, \mathrm{CdS}}$ respectively) are extracted from those plots by extrapolation of the absorption onset (straight lines), as illustrated in detail elsewhere [12]. (c,d): Valence-band photoemission spectra of CZTS (c) and CdS (d) measured at a depth where the photoemission onsets of both material are distinguishable. Plots in (c) are raw photoemission spectra, where the interface valence band maximum of CZTS $\left(E_{\mathrm{vi}, \mathrm{CZTS}}\right)$ is extracted by extrapolation with a straight line. Plots in (d) are valence band difference spectra [18] obtained from the plots in (c). From the valence band difference spectra, the interface valence band maximum of $\mathrm{CdS}\left(E_{\mathrm{vi}, \mathrm{CdS}}\right)$ is extracted by extrapolation with a straight line [19]. In all plots, blue circles refer to as-deposited samples and red triangles refer to post-annealed samples. Refer to Fig. 1 for interpreting the symbols.

in the continuous photon spectral range $0.78-3.50 \mathrm{eV}$ was used as a probe. The change in polarization upon multiple reflections at the interfaces of the CdS/CZTS/glass stack is described by the two quantities $\Psi(E)$ and $\Delta(E)$, which are measured by the ellipsometer after reflection as a function of photon energy $E$ (Fig. $1(\mathrm{a})$ ). Since $\Psi(E)$ and $\Delta(E)$ depend on the thickness of the layers and on their dielectric functions, fitting the $\Psi(E)$ and $\Delta(E)$ spectra to a parametric optical model including the layer thicknesses and their dielectric functions $\varepsilon_{1}(E), \varepsilon_{2}(E)$ as unknown parameters, the value of those unknown parameters can be determined by least-squares regression analysis [20]. Analysis and fitting of ellipsometry spectra was performed with the CompleteEase software package (version 5.06 - J.A. Woollam Co.). Further details of our measurement approach, a full list of fitted parameters, and the validity of the optical model used in this study are discussed elsewhere [12]. Note that band tails are allowed when fitting the dielectric function of CZTS, and they are in fact detected in all samples in this study (Fig. 2(a)).

X-ray photoelectron spectroscopy (XPS) was performed after the ellipsometry measurements to determine valence band offsets and to simultaneously probe the depth-dependent composition of the interface region (Fig. 1(b)). A Thermo Scientific K-Alpha instrument with a monochromated $\mathrm{Al} \mathrm{K}_{\alpha}$ x-ray source and a spot size of roughly $400 \mu \mathrm{m}$ was used. The different CZTS/CdS samples were progressively etched with low-energy $\mathrm{Ar}^{+}$ions (200 eV) from the CdS side. Photoemission spectra of the valence band region were recorded after each etching step to determine the valence band position with an analysis depth of a few nm (Fig. 1(b)). The first spectrum with a recognizable photoemission onset of CZTS is used to determine the interface valence band position of CZTS with respect to the Fermi level $\left(E_{\mathrm{vi}, \mathrm{CZTS}}\right)$. The interface valence band position of $\mathrm{CdS}\left(E_{\mathrm{vi}, \mathrm{CdS}}\right)$ is derived from the same spectrum by the technique of valence band difference spectra [18] or "direct VBO method" [7]. The interface valence band offset (VBO) is simply calculated as VBO $=E_{\mathrm{vi}, \mathrm{CdS}}-E_{\mathrm{vi}, \mathrm{CZTS}}$. The Cu 2p, Zn 2p, Sn 3d, Cd 3d and $\mathrm{S} 2 \mathrm{p}$ core levels were also recorded after each etching step to determine the depth-dependent chemical composition and correlate it to the interface electronic properties. Further details on instrument calibration and the extrapolation of valence band offsets from photoemission spectra are discussed elsewhere [12].

\section{Results}

\subsection{Interface electronic properties}

Fig. 2 shows the data from which interface valence band maxima and near-interface band gaps are extracted. Interface properties are then plotted in Fig. 3 as a function of process conditions. Some interesting observations can be made:

1. in all samples, the near-interface band gaps of both CZTS and CdS are lower than their bulk band gaps by up to $0.25 \mathrm{eV}$ and $0.16 \mathrm{eV}$ respectively (Figs. 3(a),(b)).

2. the bulk band gap of CZTS does not vary significantly from sample to sample (Fig. 3(a)). Hence, any effect of the post-annealing process and of different CdS deposition temperatures is limited to a relatively shallow region (much less than $100 \mathrm{~nm}$ ) below the interface. An exception is the as-deposited sample with a $\mathrm{CdS}$ deposition temperature of $75^{\circ} \mathrm{C}$. As will be shown later (Fig. 4), this is the only sample with a $\mathrm{Cu}$-rich composition in the CZTS bulk, which may explain the discrepancy.

3. the interface valence band maximum of CZTS (Fig. 3(c)) follows a clear trend. Both a higher 


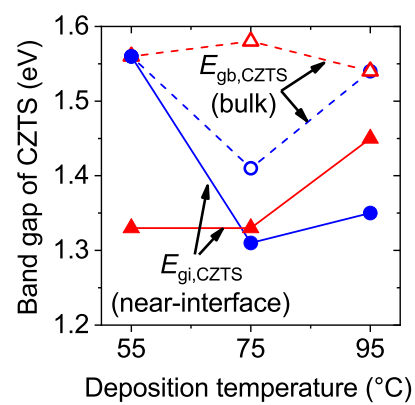

(a)

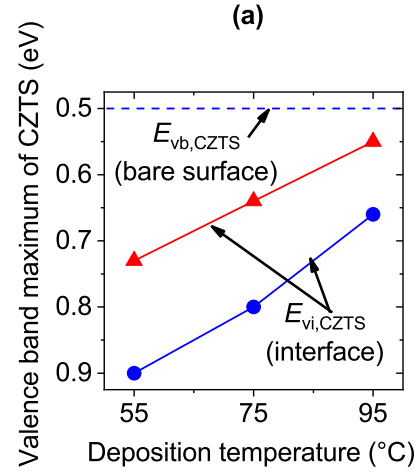

(c)

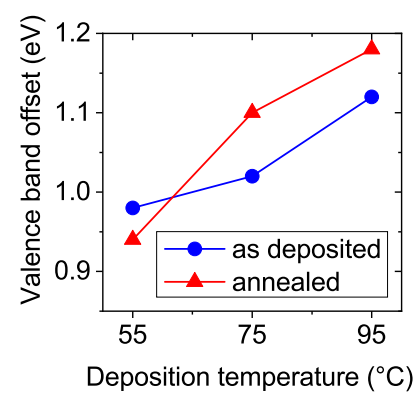

(e)

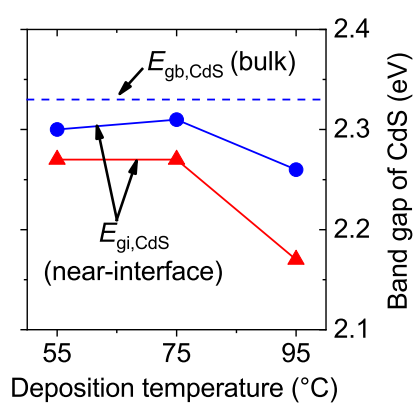

(b)

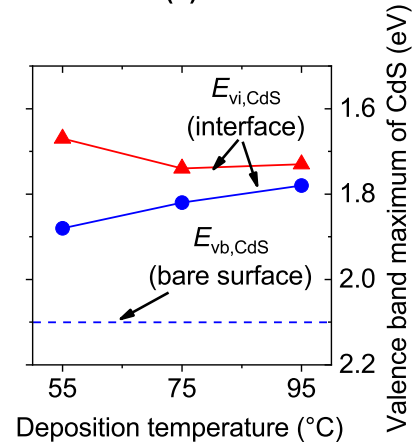

(d)

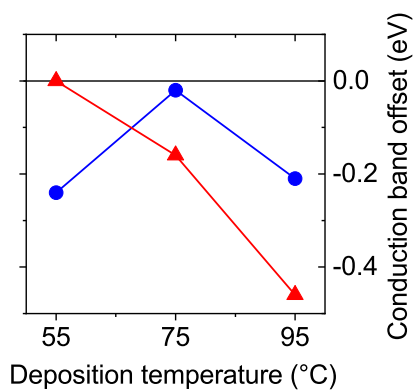

(f)
Figure 3: (a),(b): Bulk- and near-interface band gaps of CZTS $\left(E_{\mathrm{gb}, \mathrm{CZTS}}, E_{\mathrm{gi}, \mathrm{CZTS}}\right)$ and of CdS $\left(E_{\mathrm{gb}, \mathrm{CdS}}, E_{\mathrm{gi}, \mathrm{CdS}}\right)$ measured by ellipsometry. Note that $E_{\mathrm{gb}, \mathrm{CdS}}$ is obtained from a separate ellipsometry measurement on a CdS film deposited on ITO-coated glass. (c),(d): Bulk and near-interface valence band maximum of CZTS $\left(E_{\mathrm{vb}, \mathrm{CZTS}}, E_{\mathrm{vi}, \mathrm{CZTS}}\right)$ and of CdS ( $E_{\mathrm{vb}, \mathrm{CdS}}$ and $\left.E_{\mathrm{vi}, \mathrm{CdS}}\right)$ measured by XPS and expressed as energy below the Fermi level. Note that and $E_{\mathrm{vb}, \mathrm{CZTS}}$ and $E_{\mathrm{vb}, \mathrm{CdS}}$ are obtained from separate XPS measurements of a CZTS film on glass and of a CdS film on ITO-coated glass. (e): CZTS/CdS valence band offset by XPS $\left(\mathrm{VBO}=E_{\mathrm{vi}, \mathrm{CdS}}-E_{\mathrm{vi}, \mathrm{CZTS}}\right)$. (f): CZTS/CdS conduction band offset obtained by the combination of the XPS-determined VBO and the ellipsometry-determined near-interface band gaps $(\mathrm{CBO}=$ $E_{\text {gi,CdS }}-E_{\text {gi,CZTS }}-$ VBO). In all plots, blue circles (red triangles) are data points for the as-deposited (post-annealed) interfaces.

CdS deposition temperature and the post-annealing treatment contribute to raising $E_{\mathrm{vi}, \mathrm{CZTS}}$ closer to the Fermi level (more p-type), and closer to the value measured on a bare CZTS surface. This is equivalent to less band bending in the near-interface region.

4. the interface valence band maximum of CdS is weakly dependent on process conditions and is in most cases located around 1.7-1.8 eV below the Fermi level (Fig. 3(d)). Only at the lowest deposition temperature $\left(55^{\circ} \mathrm{C}\right)$ a significant difference exists between the as-deposited sample and the post-annealed sample.

5. the valence band offset becomes larger with increasing CdS deposition temperature (Fig. 3(e)).

6. the conduction band offsets of the different samples, calculated from the measured VBO and the measured interface band gaps, span over a $0.5 \mathrm{eV}$ range (Fig. 3(f)). They are in general negative or close to zero.

\subsection{Interface composition}

Some properties extracted from the XPS measurement of depth-dependent chemical composition are illustrated in Fig. 4, Fig. 5, and Fig. 6(a). The most interesting trends are:

1. all samples have roughly similar compositions in the CZTS bulk, as intended in the film growth phase (Fig. 4). An exception is the as-deposited sample with a CdS deposition temperature of $75^{\circ} \mathrm{C}$, which has a higher $\mathrm{Cu}$ content. This is also the only sample with a significantly lower CZTS bulk band gap (Fig. 3(a)), which may be ascribed to the presence of (low-band gap) copper sulfide secondary phases [21]. In fact, the bulk band gap of CZTS is found to be correlated with the average $\mathrm{Zn} / \mathrm{Cu}$ ratio in the CZTS bulk (Fig. 5(a)).

2. the Zn content steadily decreases from the CZTS bulk towards the CZTS/CdS interface in all samples, while the $\mathrm{Cu} / \mathrm{Sn}$ ratio remains roughly constant in the same region (Fig. 4). Further analysis of the $\mathrm{Zn}$, $\mathrm{Cu}$, and Sn content versus $\mathrm{Cd}$ content reveals an inverse correlation between $\mathrm{Zn}$ content and $\mathrm{Cd}$ content (Fig. 5(b)). This indicates that Cd interdiffusion into CZTS mainly results in $\mathrm{Zn}$ substitution $\left(\mathrm{Cd}_{\mathrm{Zn}}\right)$, as expected from previous studies on $\mathrm{Cd}$ incorporation in the CZTS bulk [22, 3].

3. the as-deposited samples exhibit a sharp $\mathrm{Cu}$-poor, Zn-rich region in the interface region closest to CdS, where the $\mathrm{Cu}$ - and $\mathrm{Zn}$ content appear to be inversely correlated (Fig. 4). Conversely, no sharp $\mathrm{Cu}$-poor, Zn-rich region can be identified in the post-annealed samples.

It can be interesting to determine whether a higher CdS deposition temperature and the post-annealing treatment promote interdiffusion. Interdiffusion is quantified by fitting the decay of the $\mathrm{Cd} 3 \mathrm{~d}$ core level intensity versus depth using a Fermi-Dirac-like function, i.e., $\left[1+\exp \left(x / d_{\mathrm{x}}\right)\right]^{-1}$ (details in Fig. S1, Supporting information). The fitted broadening parameter $d_{\mathrm{x}}$ of the Fermi-Dirac function is defined as the "interface grading parameter" in Fig. 6 and is taken as a measure of interdiffusion. Analysis of the interface grading parameter as a function of process conditions (Fig. 6(a)) confirms that both a higher CdS deposition temperature and a post-annealing treatment promote interdiffusion. 

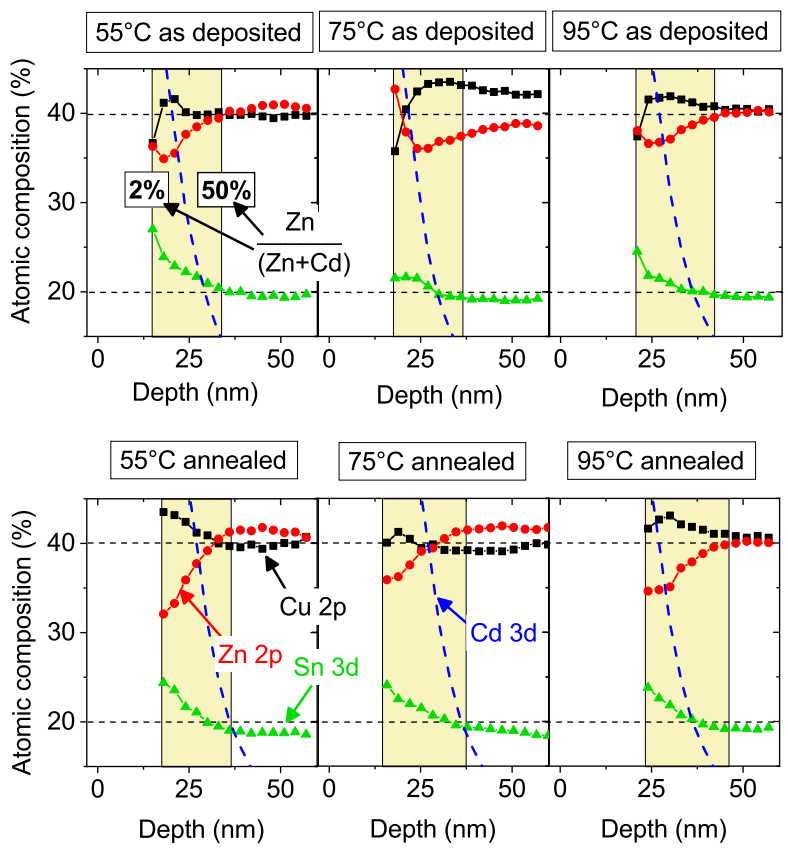

Figure 4: Depth profiles of $\mathrm{Cu}$ content (black squares), Zn content (red circles), and Sn content (green triangles) of the CdS/CZTS stacks investigated in this study. Elemental composition is derived from the relative intensities of the $\mathrm{Cu} 2 \mathrm{p}, \mathrm{Zn} 2 \mathrm{p}$, and $\mathrm{Sn} 3 \mathrm{~d}$ core level peaks measured by XPS. The sum of those three core level intensities is normalized to 1 to emphasize the relative composition of the CZTS cations. The Cd content, estimated from the Cd 3d core level peak and normalized to the total composition, is also shown for reference (dashed line). In each plot, the colored region corresponds to the depth where the $\mathrm{Zn} /(\mathrm{Zn}+\mathrm{Cd})$ ratio is between $2 \%$ and $50 \%$. The data between those two lines is considered the most representative of the region of interdiffusion. When $\mathrm{Zn} /(\mathrm{Zn}+\mathrm{Cd}) \leq 2 \%$, the relative $\mathrm{Cu}-\mathrm{Zn}$-Sn composition cannot be measured reliably due to the low core level intensity. The average $\mathrm{Zn}$ content of all samples is systematically higher than the $\mathrm{Zn}$ content measured by energy dispersive $\mathrm{X}$-ray spectroscopy (EDX) due to preferential sputtering effects, as observed by other authors [9]. For a measurement of deeper diffusion of $\mathrm{Cu}, \mathrm{Zn}$, and Sn into CdS see Fig. S2, Supporting information.

The next step is to ascertain if any of the interface electronic properties shown in Fig. 3 are directly related to interdiffusion. The interface valence band maximum of CZTS is the property with the strongest correlation to the interface grading parameter, with higher-lying valence band maxima (less band bending) corresponding to more interdiffusion (Fig. 6(b)). The near-interface band gap of CZTS does not appear to be correlated to interdiffusion (Fig. 6(c)), whereas the near-interface band gap of CdS has a weak, negative correlation to interdiffusion (Fig. 6(d)).

\section{Discussion}

\subsection{Near-interface band gaps}

The causes of near-interface band gap narrowing of CZTS and CdS are not obvious from the present experimental data. Nevertheless, there is some evidence that Cd interdiffusion is partially responsible for near-interface band gap narrowing in CZTS. As noted above, Fig. 5(b)

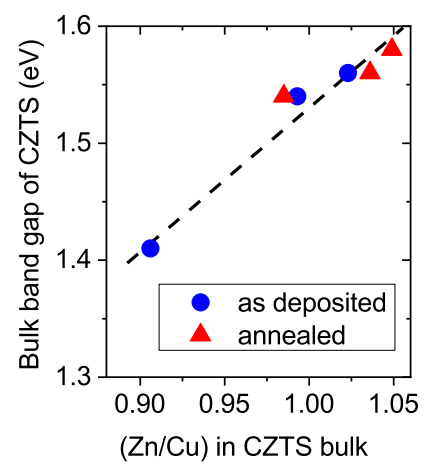

(a)

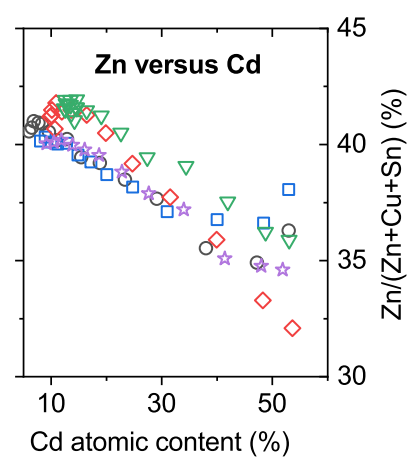

(b)
Figure 5: (a): Correlation between the bulk band gap of CZTS $\left(E_{\mathrm{gb}, \mathrm{CZTS}}\right)$ and the average $\mathrm{Zn} / \mathrm{Cu}$ ratio measured by XPS in the CZTS bulk, defined as the region where $\mathrm{Zn} /(\mathrm{Zn}+\mathrm{Cd})>50 \%$, to the right of the colored area in Fig. 4. The dashed line is a guide to the eye. (b): Correlation between the Zn content in CZTS and the total Cd content. Each data point corresponds to a certain depth in the XPS measurement of Fig. 1(b). Different symbols correspond to different samples. Circles: $55^{\circ} \mathrm{C}$ as deposited; Diamonds: $55^{\circ} \mathrm{C}$ annealed; Triangles: $75^{\circ} \mathrm{C}$ annealed; Squares: $95^{\circ} \mathrm{C}$ as deposited; Stars: $95^{\circ} \mathrm{C}$ annealed. Data from the $\mathrm{Cu}$-rich sample $\left(75^{\circ} \mathrm{C}\right.$ as deposited) follows roughly the same trends but is omitted for better readability.

reveals an inverse correlation between the total $\mathrm{Cd}$ content and the $\mathrm{Zn} /(\mathrm{Zn}+\mathrm{Cu}+\mathrm{Sn})$ content in the region of interdiffusion. This indicates that $\mathrm{Cd}$ preferentially replaces $\mathrm{Zn}$, in agreement with previous studies on Cd incorporation into CZTS [23, 22, 3, 7]. The band gap of bulk $\mathrm{Cu}_{2} \mathrm{Zn}_{1-x} \mathrm{Cd}_{x} \mathrm{SnS}_{4}$ was previously found to decrease from $1.54 \mathrm{eV}$ to $1.36 \mathrm{eV}$ as $x$ was intentionally changed from 0 to 0.5 in the bulk [22]. Such band gap changes by $\mathrm{Cd}$ incorporation are consistent with the $E_{\text {gi,CZTS }}$ values measured in this study (Fig. 3(a)). If the parameter $x$ were the only factor affecting the near-interface band gap of CZTS in our study, the measured $E_{\text {gi,CzTs }}$ values would imply that $\mathrm{Cd}$ substitutes at least $50 \%$ of $\mathrm{Zn}$ in the near-interface region under most process conditions. However, the $\mathrm{Zn}$ content measured by XPS decreases by much less than a factor 2 from bulk CZTS to the interface (Fig. 4). Furthermore, $E_{\text {gi,CZTS }}$ does not show a clear inverse correlation to the interface grading parameter (Fig. 6(c)). We therefore conclude that other unidentified band gap narrowing mechanisms must coexist. They could be related, for example, to the presence of low-band gap secondary phases or to increasing cation disorder near the interface [24]. Both effects can be detected by the ellipsometry measurement and could therefore contribute to the experimentally observed band gap narrowing in CZTS. Conversely, other band gap narrowing mechanisms that involve extremely thin (1-2 nm) interface layers, such as those due to unpassivated surface states [5], cannot be resolved by the optical model in Fig. 1(a). If present, they would cause even stronger band gap narrowing than the values reported in this study. Regardless of the cause, near-interface band gap narrowing in CZTS is likely to have negative conse- 


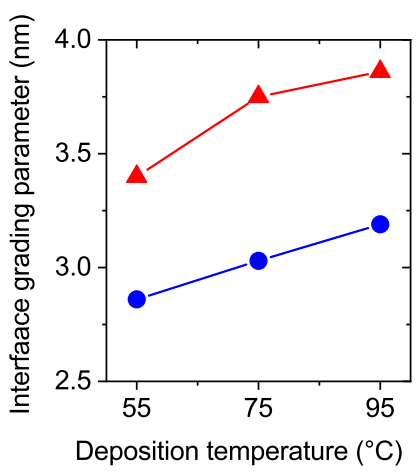

(a)

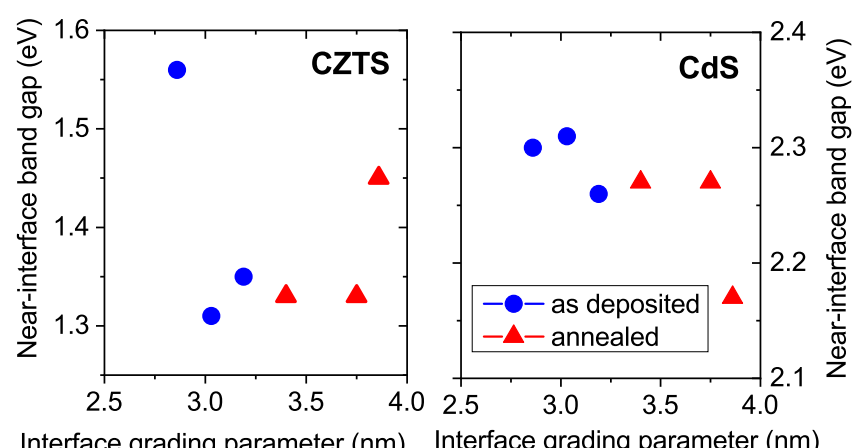

(c)

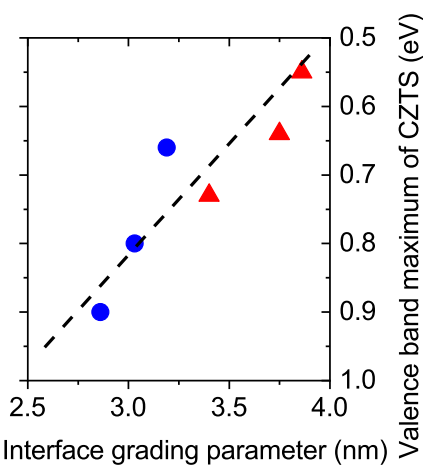

(b)

(d)
Figure 6: (a): Grading parameter of the CZTS/CdS interface as a function of process conditions. (b): Correlation between the interface grading parameter and the interface valence band maximum of CZTS $\left(E_{\mathrm{vi}, \mathrm{CZTS}}\right)$. The dashed line is a guide to the eye. (c): Correlation between the interface grading parameter and the near-interface band gap of CZTS $\left(E_{\text {gi,CZTS }}\right)$. (d): Correlation between the interface grading parameter and the near-interface band gap of CdS $\left(E_{\mathrm{gi}, \mathrm{CdS}}\right)$.

quences on the open circuit voltage of the solar cell and can explain the outcome of temperature-dependent open circuit voltage measurements [5]. We note that near-interface band gap narrowing in CZTS is the opposite effect of the (beneficial) near-interface band gap widening in the similar material $\mathrm{Cu}(\mathrm{In}, \mathrm{Ga}) \mathrm{Se}_{2}$ [25]. This discrepancy may explain why CZTS solar cells appear to be limited by interface recombination, whereas $\mathrm{Cu}(\mathrm{In}, \mathrm{Ga}) \mathrm{Se}_{2}$ solar cells are not [7].

Near-interface band gap narrowing in CdS is not readily explained by qualitative arguments. Several authors, including some of the authors of the present article, had previously speculated that the CdS band gap may increase near the interface with CZTS due to preferential Zn interdiffusion $[6,7]$. The argument was that the band gap of the resulting $\mathrm{Zn}_{x} \mathrm{Cd}_{1-x} \mathrm{~S}$ interface phase would increase with increasing $x$. However, under all process conditions examined in this study, $E_{\text {gi,CdS }}$ is experimentally found to decrease with respect to $E_{\mathrm{gb}, \mathrm{CdS}}$ instead (Fig. 3(b)). The band gap decrease is stronger with stronger interdiffusion (Fig. 6(d)). We note that band gap narrowing in CdS could also be inferred by inspecting the absorption onset of $\mathrm{CdS}$ in quantum efficiency spectra of finished CZTS/CdS solar cells. Onsets at lower photon ener- gies have indeed been observed in the presence of a postannealing treatment [26]. While relatively deep, preferential $\mathrm{Zn}$ diffusion into $\mathrm{CdS}$ is observed in some of our samples (Fig. S2, Supporting information), the detected $\mathrm{Zn}$ content in $\mathrm{CdS}$ is never above $1 \%$ and does not have a clear correlation with process conditions. Even though preferential Zn interdiffusion has been reported by many authors, an interface phase with significant mixing of $\mathrm{Zn}$ and Cd could only be detected at the CZTSe/CdS interface but not at the CZTS/CdS interface [27]. Interestingly, two independent studies [28, 29] found a narrowed near-interface band gap of $2.2 \mathrm{eV}$ in $\mathrm{CdS}$ after interface formation with $\mathrm{Cu}_{2} \mathrm{ZnSn}\left(\mathrm{S}_{0.28} \mathrm{Se}_{0.72}\right)_{4}$ and with $\mathrm{CuInSe}{ }_{2}$, similarly to what is found in the present study. They both measured the near-interface band gap by a combination of direct- and inverse photoemission spectroscopy on a thin CdS layer grown on the absorber material. Band gap narrowing was in those cases attributed to Se interdiffusion. However, our study shows similar $E_{\text {gi,CdS values for } \mathrm{CdS}}$ deposited on a Se-free CZTS absorber using a significantly different characterization technique. One possible explanation for the decreasing CdS band gap with increasing processing temperatures is the increase in grain size of the CdS film with temperature [17]. It is then possible that the band gap of the films processed at low temperatures is higher due to quantum confinement effects arising from the very small grain size [7]. Such effects have been observed in chemically deposited films with grain sizes of a few nm [17]. An alternative explanation could be that the increase of $\mathrm{Cu}$ content at the interface upon a postannealing treatment (Fig. 4) results in CdS band gap narrowing over a very thin interface region. As noted previously, very thin interface regions are difficult to resolve by ellipsometry due to parameter correlation in the data fitting phase [12]. Therefore the measured CdS band gap may be an average band gap over the CdS layer thickness. In the sample fabrication phase, the CdS thickness was intentionally kept low in order to reduce such errors [12].

\subsection{Band bending and valence band offsets}

The position of the interface valence band maximum of CZTS, related to interface band bending, is clearly correlated to the interface grading parameter, i.e., to interdiffusion (Fig. 6(b)). If near-interface band gap narrowing in CZTS was caused by a valence band up-shift, it could also contribute to a decrease in $E_{\mathrm{vi}, \mathrm{CZTS}}$ (smaller apparent band bending). However, $E_{\mathrm{vi}, \mathrm{CZTS}}$ and $E_{\text {gi,CZTS }}$ are not correlated (Fig. 3(a,c)) so we expect this to be a minor effect. As discussed in the above sections, interdiffusion most likely results in $\mathrm{Cd}_{\mathrm{Zn}}$ substitutional defects in CZTS (Fig. 5(b)). An inverse correlation can then be inferred between downward band bending in CZTS and the density of $\mathrm{Cd}_{\mathrm{Zn}}$ defects near the interface. Importantly, the $\mathrm{Cd}_{\mathrm{Zn}}$ defect is known to slightly decrease the intrinsic p-type doping of CZTS in the bulk [3]. This would imply that enhanced interdiffusion would correspond to larger downward band bending - exactly the opposite of the experi- 


\begin{tabular}{|c|c|c|c|c|c|c|c|c|c|c|}
\hline $\begin{array}{c}\text { CdS deposition } \mathrm{T} \\
\left({ }^{\circ} \mathrm{C}\right)\end{array}$ & $\begin{array}{l}E_{\mathrm{vb}, \mathrm{CZTS}} \\
(\mathrm{eV})\end{array}$ & $\begin{array}{l}E_{\mathrm{vi}, \mathrm{CZTS}} \\
(\mathrm{eV})\end{array}$ & $\begin{array}{l}E_{\mathrm{vb}, \mathrm{CdS}} \\
(\mathrm{eV})\end{array}$ & $\begin{array}{l}E_{\mathrm{vi}, \mathrm{CdS}} \\
(\mathrm{eV})\end{array}$ & $\begin{array}{l}\mathrm{VBO} \\
(\mathrm{eV}) \\
\end{array}$ & $\begin{array}{l}E_{\mathrm{gb}, \mathrm{CZTS}} \\
(\mathrm{eV})\end{array}$ & $\begin{array}{l}E_{\text {gi,CZTS }} \\
(\mathrm{eV})\end{array}$ & $\begin{array}{l}E_{\mathrm{gb}, \mathrm{CdS}} \\
(\mathrm{eV})\end{array}$ & $\begin{array}{l}E_{\text {gi, CdS }} \\
(\mathrm{eV})\end{array}$ & $\begin{array}{l}\text { CBO } \\
(\mathrm{eV})\end{array}$ \\
\hline \multicolumn{11}{|l|}{ as deposited CdS } \\
\hline 55 & 0.50 & 0.90 & 2.10 & 1.88 & 0.98 & 1.56 & 1.56 & 2.33 & 2.30 & -0.24 \\
\hline 75 & $"$ & 0.80 & $"$ & 1.82 & 1.02 & 1.41 & 1.31 & $"$ & 2.31 & -0.02 \\
\hline 95 & $"$ & 0.66 & $"$ & 1.78 & 1.12 & 1.54 & 1.35 & $"$ & 2.26 & -0.21 \\
\hline \multicolumn{11}{|l|}{ post-annealed CdS } \\
\hline 55 & 0.50 & 0.73 & 2.10 & 1.67 & 0.94 & 1.56 & 1.33 & 2.33 & 2.27 & +0.00 \\
\hline 75 & $"$ & 0.64 & $"$ & 1.74 & 1.10 & 1.58 & 1.33 & $"$ & 2.27 & -0.16 \\
\hline 95 & $"$ & 0.55 & $"$ & 1.73 & 1.18 & 1.54 & 1.45 & $"$ & 2.17 & -0.46 \\
\hline
\end{tabular}

Table 1: Numerical values of the quantities measured in this work, which are also plotted in Fig. 7. See Fig. 1(c) for a key to the symbols.
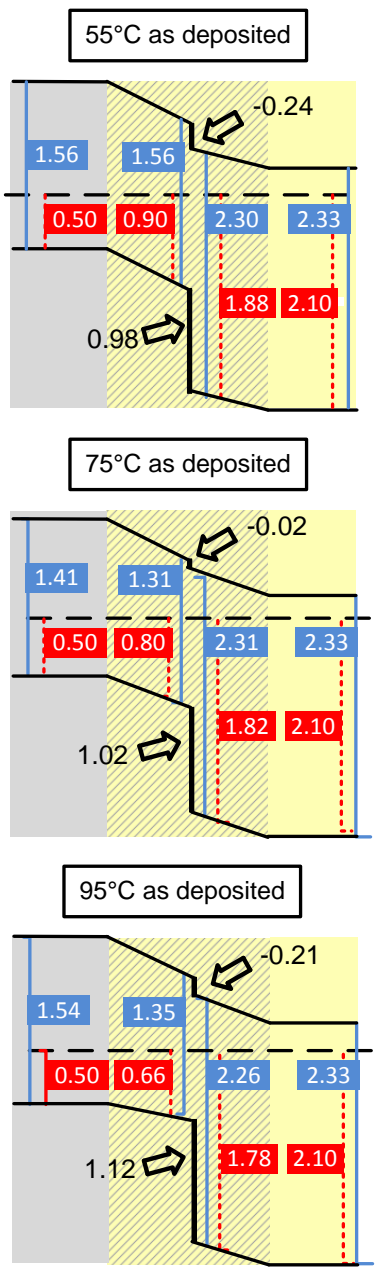

Figure 7: Interface band diagrams of the CZTS/CdS interfaces studied in this work. See Fig. 1(c) for a key to the symbols and colors.

mentally observed effect. It is then likely that the presence of the $\mathrm{Cd}_{\mathrm{Zn}}$ defect is coupled with the presence of other defects that influence the Fermi level position near the interface [7]. To attempt identification of such defects, we return to our previous observation of sharp, Cu-poor Zn-rich interfaces in the as-deposited samples as opposed to the post-annealed samples (Fig. 4). Due to those differences in composition, a higher density of $\mathrm{Zn}_{\mathrm{Cu}}$ donors, $\mathrm{Cd}_{\mathrm{Cu}}$ donors, and $\left(\mathrm{Cd}_{\mathrm{Cu}}+\mathrm{V}_{\mathrm{Cu}}\right)$ neutral clusters may be expected in the as-deposited samples [23]. On the other hand, the post-annealed samples are likely to have a higher density of $\mathrm{Cu}_{\mathrm{zn}}$ acceptors that are not compensated by $\mathrm{Zn}_{\mathrm{Cu}}$ donors due to their higher interface $\mathrm{Cu} / \mathrm{Zn}$ ratio. In all the above cases, larger band bending is expected in the as-deposited samples as observed experimentally and as argued in the following. First, near-interface donors $\left(\mathrm{Zn}_{\mathrm{Cu}}\right.$ or $\mathrm{Cd}_{\mathrm{Cu}}$ ) would make the near-interface region more ntype or even pin the Fermi level to the donor level close to the conduction band (large band bending) [7]. Secondly, neutral $\left(\mathrm{Cd}_{\mathrm{Cu}}+\mathrm{V}_{\mathrm{Cu}}\right)$ clusters would remove some of the shallow $\mathrm{V}_{\mathrm{Cu}}$ acceptors which are believed to be responsible for p-type conductivity in CZTS [30]. Finally, uncompensated $\mathrm{Cu}_{\mathrm{Zn}}$ acceptors may pin the Fermi level to the $\mathrm{Cu}_{\mathrm{Zn}}$ level close to the valence band and thus prevent band bending. Hence, we propose that the post-annealing treatment changes the near-interface defect chemistry by removing the $\mathrm{Cu}$-poor, Zn-rich composition of as-deposited interfaces and preventing large band bending in CZTS. This effect is detrimental both for effective electron-hole separation and for the achievement of low electron-hole recombination rates at the interface $[31,32]$. Increasing the CdS deposition temperature similarly appears to reduce band bending in CTZS (Fig. 3(c)).

The dependence of $E_{\mathrm{vi}, \mathrm{CdS}}$ on process parameters (Fig. 3(d)) is weaker than the dependence of $E_{\mathrm{vi}, \mathrm{CZTS}}$ on process parameters, which results in significant variations in the VBO from sample to sample (Fig. 3(e)). Only at a $\mathrm{CdS}$ deposition temperature of $55^{\circ} \mathrm{C}$ does the $\mathrm{VBO}$ remain roughly constant before and after post-annealing. Conversely, at higher deposition temperatures the interface valence band maximum of $\mathrm{CdS}$ appears to be pinned around 1.7-1.8 eV below the Fermi level (Fig. 3(d)). Since Fermi level pinning on both sides of the interface is necessary for altering the band offsets [7], we conclude that the Fermi level of CZTS is pinned closer and closer to the valence band (less downward band bending) with both increasing CdS deposition temperature and post-annealing, and that the Fermi level of CdS is pinned at around 1.7$1.8 \mathrm{eV}$ above the valence band when the $\mathrm{CdS}$ deposition temperature is $75^{\circ} \mathrm{C}$ or above.

\subsection{Conduction band offsets}

The conduction band offset is a very important property of CZTS/CdS interfaces. A negative CBO decreases the barrier for recombination at the interface and negatively 


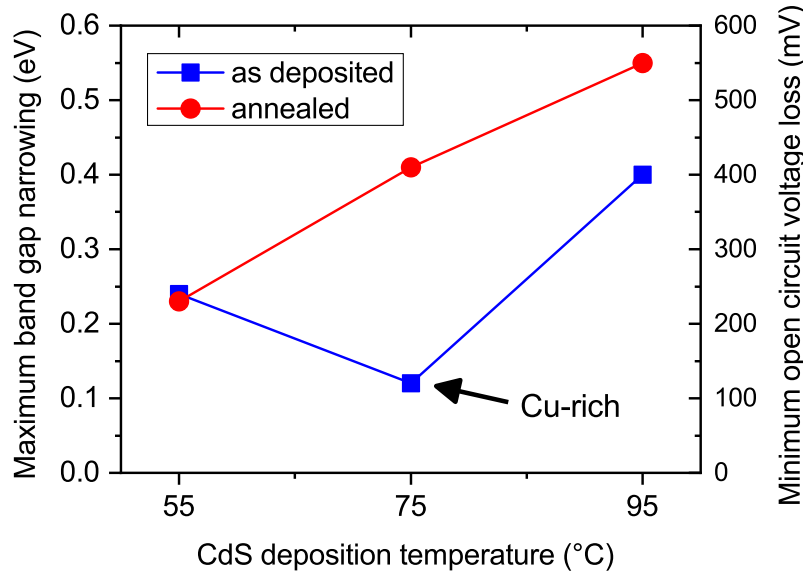

Figure 8: Maximum band gap narrowing in CZTS/CdS stacks relative to the bulk CZTS band gap $E_{\mathrm{gb}, \mathrm{CZTS}}$. If the CBO is positive, the maximum band gap narrowing corresponds to $\left(E_{\mathrm{gb}, \mathrm{CZTS}}-\right.$ $\left.E_{\mathrm{gi}, \mathrm{CZTS}}\right)$. If the $\mathrm{CBO}$ is negative, it corresponds to $\left(E_{\mathrm{gb}, \mathrm{CZTS}}-\right.$ $\left.E_{\mathrm{gi}, \mathrm{CZTS}}-\mathrm{CBO}\right)$ to include the effect of cross-recombination at the interface from the CdS conduction band to the CZTS valence band. Assuming that the dominant recombination path in a CZTS solar cell is in the regions with a narrowed band gap, the maximum band gap narrowing is equal to the minimum loss in open circuit voltage to be expected compared to an ideal solar absorber with uniform band gap $E_{\mathrm{gb}, \mathrm{CZTS}}$.

affects the open circuit voltage of the solar cell. A large positive CBO blocks electron transport across the interface and negatively affects either the short circuit current or the fill factor of the solar cell. In this study, the CBO follows roughly the reverse trend of the VBO, even though differences in the near-interface band gaps of the two materials modify the conclusions that would be drawn by assuming that bulk band gaps are equal to near-interface band gaps. The interface with $55^{\circ} \mathrm{C} \mathrm{CdS}$ deposition temperature and no post-annealing can be considered as the one that is least influenced by process-related non-idealities, since the near-interface band gaps of CZTS and CdS are nearly equal to the bulk band gaps, and relatively large band bending occurs in CZTS as expected by p-n junction theory. The $\mathrm{CBO}$ in that sample is $-0.24 \mathrm{eV}$ (Fig. 3(f)), which is lower than optimal and may enhance interface recombination. For the sample deposited at $95^{\circ} \mathrm{C}$ and postannealed, near-interface band gap narrowing is stronger in CdS than in CZTS, which results in a very large negative $\mathrm{CBO}(-0.46 \mathrm{eV})$. For all other samples, near-interface band gap narrowing is stronger in CZTS than in CdS, which results in less negative CBOs than what would be expected by simply adding the bulk band gaps to the VBO (Fig. 3(f)). This indicates that an optimal CBO can be achieved by tuning the process conditions that have an influence on chemical interdiffusion. The CBO increase upon $\mathrm{Cd}$ incorporation into CZTS is consistent with previous studies [33].

\subsection{Optimal processing of CZTS/CdS interfaces}

The interface band diagrams derived from the combined XPS/ellipsometry measurement are plotted in Fig. 7 . They show that all the investigated process conditions result in some non-optimal features. Either a negative, clifflike CBO or a strongly narrowed interface band gap (or a combination of both) are present in all cases (Fig. 7). Both effects are detrimental for the open circuit voltage of a solar cell, although they may be difficult to distinguish from each other in the device characterization phase [5]. The least harmful process conditions may be identified by calculating the maximum narrowing in the energy barrier for electron-hole recombination relative to the bulk CZTS band gap in each sample. If the $\mathrm{CBO}$ is positive, this simply corresponds to $\left(E_{\mathrm{gb}, \mathrm{CZTS}}-E_{\mathrm{gi}, \mathrm{CZTS}}\right)$. If the CBO is negative, this corresponds to $\left(E_{\mathrm{gb}, \mathrm{CZTS}}-E_{\mathrm{gi}, \mathrm{CZTS}}-\mathrm{CBO}\right)$. The calculated values, shown in Fig. 8 , give an idea of the minimum open circuit voltage loss to be expected in CZTS/CdS solar cells dominated by interface- or nearinterface recombination. From Fig. 8, a low CdS deposition temperature $\left(55^{\circ} \mathrm{C}\right)$ appears as the least harmful processing route. Interestingly, the post-annealing treatment on CdS deposited at $55^{\circ} \mathrm{C}$ is beneficial in some aspects (increase of the $\mathrm{CBO}$ ) but detrimental in other aspects (nearinterface band gap narrowing in CZTS and smaller band bending). The detrimental effect of smaller band bending cannot be included in Fig. 8 because it does not involve band gap changes. As the as-deposited interfaces consistently achieve larger band bending, we conclude that the least unfavorable process conditions consist of a CdS deposition temperature of $55^{\circ} \mathrm{C}$ without post-annealing. Even in that case, a minimum open circuit voltage loss of about $230 \mathrm{mV}$ compared to the Shockley-Queisser limit [34] is still expected (Fig. 8). Although the sample with $\mathrm{Cu}-$ rich CZTS has the lowest expected open circuit voltage loss among all samples (Fig. 8), Cu-rich CZTS has very poor photovoltaic performance due to its unfavorable bulk defect chemistry and secondary phases [21, 30]. Furthermore, the small near-interface band gap decrease of that sample may simply be due to its band gap already being low in the bulk (Fig. 3(a)). We emphasize that only selected properties have been measured in this article and have been considered as indicators of the interface quality. It is still perfectly possible that other interface properties (density of deep traps, epitaxial interface, crystalline quality of CdS) improve upon post-annealing, and even outweigh the detrimental effects shown in this paper. In fact, improved solar cell efficiency has been reported by some authors when incorporating a post-annealing step after interface formation $[6,35]$. Unfortunately, the conclusions of this study cannot be correlated to device performance, because the CZTS/CdS films for ellipsometry analysis had to be deposited directly on glass. Including the standard back contact (Mo) in the device structure results in two additional layers (Mo and $\mathrm{MoS}_{2}$ ), with an unacceptable increase in the number of parameters needed to fit the ellipsometry spectra [11]. 


\section{Conclusion}

We have applied a combination of photoemission spectroscopy and spectroscopic ellipsometry to study the electronic properties of the CZTS/CdS interface as a function of process conditions. The interface properties were unfavorable in all cases, either due to a cliff-like conduction band offset, or to substantial band gap narrowing of CZTS near the interface, or to both effects. Interface band gap narrowing of both CZTS and CdS was found to be somewhat correlated with chemical interdiffusion across the interface, but identification of the specific band gapnarrowing mechanisms will require a dedicated experimental study. A higher CdS deposition temperature and a CdS post-annealing step were both found to enhance interdiffusion. Even though interdiffusion can optimize the conduction band offset of the interface under some process conditions, this comes at the cost of near-interface band gap narrowing and smaller band bending in CZTS, which are detrimental effects. We conclude that a low CdS deposition temperature of $55^{\circ} \mathrm{C}$, without post-annealing, appears as the processing route that is least harmful for the interface properties measured in this study. Even under such conditions, a minimum open circuit voltage loss of $230 \mathrm{mV}$ is expected due to interface- or near-interface recombination. A carefully selected replacement material for CdS may alleviate the $\mathrm{CBO}$ problem as well as the $\mathrm{Cd}$ interdiffusion problem believed to cause band gap narrowing in CZTS. Intentional surface oxidation of CZTS may also change the interface properties significantly compared to the samples investigated in this study.

\section{Acknowledgement}

This work was supported by the Danish Council for Strategic Research, VILLUM Fonden (grant 9455) and the Innovation Fund Denmark (File No. 5016-00102).

\section{References}

[1] T. Ericson, F. Larsson, T. Törndahl, C. Frisk, J. Larsen, V. Kosyak, C. Hägglund, S. Li, C. Platzer-Björkman, Zinc-TinOxide Buffer Layer and Low Temperature Post Annealing Resulting in a $9.0 \%$ Efficient Cd-Free $\mathrm{Cu}_{2} \mathrm{ZnSnS}_{4}$ Solar Cell, Solar RRL 1 (5) (2017) 1700001. doi:10.1002/solr.201700001.

[2] S. Tajima, T. Itoh, H. Hazama, K. Ohishi, R. Asahi, Improvement of the open-circuit voltage of $\mathrm{Cu}_{2} \mathrm{ZnSnS}_{4}$ solar cells using a two-layer structure, Applied Physics Express 8 (082302) (2015) 1-4. doi:10.7567/APEX.8.082302.

[3] C. Yan, K. Sun, J. Huang, S. Johnston, F. Liu, B. P. Veettil, K. Sun, A. Pu, F. Zhou, J. A. Stride, M. A. Green, X. Hao, Beyond $11 \%$ Efficient Sulfide Kesterite $\mathrm{Cu}_{2} \mathrm{Zn}_{1} \mathrm{Cd}_{1-x} \mathrm{SnS}_{4}$ Solar Cell: Effects of Cadmium Alloying, ACS Energy Letters 2 (4) (2017) 930-936. doi:10.1021/acsenergylett.7b00129.

[4] R. Scheer, Activation energy of heterojunction diode currents in the limit of interface recombination, Journal of Applied Physics 105 (10) (2009) 104505. doi:10.1063/1.3126523.

[5] A. Crovetto, M. Palsgaard, T. Gunst, T. Markussen, K. Stokbro, M. Brandbyge, O. Hansen, Interface band gap narrowing behind open circuit voltage losses in $\mathrm{Cu}_{2} \mathrm{ZnSnS}_{4}$ solar cells, Applied Physics Letters 110 (8) (2017) 083903. doi:10.1063/1.4976830.
[6] S. Tajima, M. Umehara, M. Hasegawa, T. Mise, T. Itoh, $\mathrm{Cu}_{2} \mathrm{ZnSnS}_{4}$ photovoltaic cell with improved efficiency fabricated by high-temperature annealing after CdS buffer-layer deposition, Progress in Photovoltaics: Research and Applications 25 (1) (2017) 14-22. doi:10.1002/pip.2837.

[7] A. Crovetto, O. Hansen, What is the band alignment of $\mathrm{Cu}_{2} \mathrm{ZnSn}(\mathrm{S}, \mathrm{Se})_{4}$ solar cells?, Solar Energy Materials and Solar Cells 169 (2017) 177-194. doi:10.1016/j.solmat.2017.05.008.

[8] P. D. Antunez, D. M. Bishop, Y. Luo, R. Haight, Efficient kesterite solar cells with high open-circuit voltage for applications in powering distributed devices, Nature Energydoi:10.1038/s41560-017-0028-5.

[9] K. Sun, C. Yan, F. Liu, J. Huang, F. Zhou, J. A. Stride, M. Green, X. Hao, Over 9\% Efficient Kesterite $\mathrm{Cu}_{2} \mathrm{ZnSnS}_{4}$ Solar Cell Fabricated by Using $\mathrm{Zn}_{1-x} \mathrm{Cd}_{x} \mathrm{~S}$ Buffer Layer, Advanced Energy Materials 6 (1600046) (2016) 1-6. doi:10.1002/aenm.201600046.

[10] M. Bär, B.-A. Schubert, B. Marsen, R. G. Wilks, S. Pookpanratana, M. Blum, S. Krause, T. Unold, W. Yang, L. Weinhardt, C. Heske, H.-W. Schock, Cliff-like conduction band offset and $\mathrm{KCN}$-induced recombination barrier enhancement at the $\mathrm{CdS} / \mathrm{Cu}_{2} \mathrm{ZnSnS}_{4}$ thin-film solar cell heterojunction, Applied Physics Letters 99 (22) (2011) 222105. doi:10.1063/1.3663327.

[11] A. Crovetto, R. Chen, R. B. Ettlinger, A. C. Cazzaniga, J. Schou, C. Persson, O. Hansen, Dielectric function and double absorption onset of monoclinic $\mathrm{Cu}_{2} \mathrm{SnS}_{3}$ : Origin of experimental features explained by first-principles calculations, Solar Energy Materials and Solar Cells 154 (2016) 121-129. doi:10.1016/j.solmat.2016.04.028.

[12] A. Crovetto, Measuring complete band diagrams of non-ideal heterointerfaces by combining ellipsometry and photoemission spectroscopy, ArXiv:1804.05424arXiv:/arxiv.org/abs/1804.05424.

[13] A. Cazzaniga, A. Crovetto, C. Yan, K. Sun, X. Hao, J. Ramis Estelrich, S. Canulescu, E. Stamate, N. Pryds, O. Hansen, J. Schou, Ultra-thin $\mathrm{Cu}_{2} \mathrm{ZnSnS}_{4}$ solar cell by pulsed laser deposition, Solar Energy Materials and Solar Cells 166 (2017) 91-99. doi:10.1016/j.solmat.2017.03.002.

[14] J. Li, H. Du, J. Yarbrough, A. Norman, K. Jones, G. Teeter, F. L. Terry, D. Levi, Spectral optical properties of $\mathrm{Cu}_{2} \mathrm{ZnSnS}_{4}$ thin film between 0.73 and $6.5 \mathrm{eV}$., Optics express $20 \mathrm{Suppl}$ 2 (102) (2012) A327-32. doi:10.1364/OE.20.00A327.

[15] A. Crovetto, A. Cazzaniga, R. B. Ettlinger, J. Schou, O. Hansen, Optical properties and surface characterization of pulsed laser-deposited $\mathrm{Cu}_{2} \mathrm{ZnSnS}_{4}$ by spectroscopic ellipsometry, Thin Solid Films 582 (2015) 203-207. doi:10.1016/j.tsf.2014.11.075.

[16] T. Gershon, B. Shin, N. Bojarczuk, M. Hopstaken, D. B. Mitzi, S. Guha, The Role of Sodium as a Surfactant and Suppressor of Non-Radiative Recombination at Internal Surfaces in $\mathrm{Cu}_{2} \mathrm{ZnSnS}_{4}$, Advanced Energy Materials 5 (2) (2014) 1400849. doi:10.1002/aenm.201400849.

[17] G. Hodes, Chemical solution deposition of semiconductor films, Marcel Dekker, New York, Basel, 2002.

[18] A. Klein, T. Löher, Y. Tomm, C. Pettenkofer, W. Jaegermann, Band lineup between CdS and ultra high vacuum-cleaved $\mathrm{CuInS}_{2}$ single crystals, Applied Physics Letters 70 (10) (1997) 1299-1301. doi:10.1063/1.118517.

[19] A. Crovetto, C. Yan, B. Iandolo, F. Zhou, J. Stride, J. Schou, X. Hao, O. Hansen, Lattice-matched $\mathrm{Cu}_{2} \mathrm{ZnSnS}_{4} / \mathrm{CeO}_{2}$ solar cell with open circuit voltage boost, Applied Physics Letters 109 (23) (2016) 233904. doi:10.1063/1.4971779.

[20] H. Fujiwara, Spectroscopic Ellipsometry - Principles and Applications, Wiley, 2007.

[21] S. Siebentritt, Why are kesterite solar cells not $20 \%$ efficient?, Thin Solid Films 535 (2013) 1-4.

[22] Z. Su, J. M. R. Tan, X. Li, X. Zeng, S. K. Batabyal, L. H. Wong, Cation Substitution of Solution-Processed $\mathrm{Cu}_{2} \mathrm{ZnSnS}_{4}$ Thin Film Solar Cell with over 9\% Efficiency, Advanced Energy Materials 5 (19) (2015) 1500682. doi:10.1002/aenm.201500682.

[23] T. Maeda, S. Nakamura, T. Wada, First-Principles Study 
on $\mathrm{Cd}$ Doping in $\mathrm{Cu}_{2} \mathrm{ZnSnS}_{4}$ and $\mathrm{Cu}_{2} \mathrm{ZnSnSe}_{4}$, Japanese Journal of Applied Physics 51 (10S) (2012) 10NC11. doi:10.1143/JJAP.51.10NC11.

[24] M. Valentini, C. Malerba, F. Menchini, D. Tedeschi, A. Polimeni, M. Capizzi, A. Mittiga, Effect of the order-disorder transition on the optical properties of $\mathrm{Cu}_{2} \mathrm{ZnSnS}_{4}$, Applied Physics Letters 108 (21) (2016) 211909. doi:10.1063/1.4952973.

[25] C. Heske, D. Eich, R. Fink, E. Umbach, T. van Buuren, C. Bostedt, L. J. Terminello, S. Kakar, M. M. Grush, T. A. Callcott, F. J. Himpsel, D. L. Ederer, R. C. C. Perera, W. Riedl, F. Karg, Observation of intermixing at the buried $\mathrm{CdS} / \mathrm{Cu}(\mathrm{In}$, Ga) $\mathrm{Se}_{2}$ thin film solar cell heterojunction, Applied Physics Letters 74 (10) (1999) 1451. doi:10.1063/1.123578.

[26] C. Yan et al., to be published.

[27] M. Bär, I. Repins, L. Weinhardt, J.-H. Alsmeier, S. Pookpanratana, M. Blum, W. Yang, C. Heske, R. G. Wilks, R. Noufi, ZnSe-Cd-S Interlayer Formation at the $\mathrm{CdS} / \mathrm{Cu}_{2} \mathrm{ZnSnSe}_{4}$ ThinFilm Solar Cell Interface, ACS Energy Letters 2 (2017) 16321640. doi:10.1021/acsenergylett.7b00140.

[28] N. Terada, S. Yoshimoto, K. Chochi, T. Fukuyama, M. Mitsunaga, H. Tampo, H. Shibata, K. Matsubara, S. Niki, N. Sakai, T. Katou, H. Sugimoto, Characterization of electronic structure of $\mathrm{Cu}_{2} \mathrm{ZnSn}\left(\mathrm{S}_{x} \mathrm{Se}_{1-x}\right)_{4}$ absorber layer and $\mathrm{CdS} / \mathrm{Cu}_{2} \mathrm{ZnSn}\left(\mathrm{S}_{x} \mathrm{Se}_{1-x}\right)_{4}$ interfaces by in-situ photoemission and inverse photoemission spectroscopies, Thin Solid Films 582 (2015) 166-170. doi:10.1016/j.tsf.2014.09.037.

[29] M. Morkel, L. Weinhardt, B. Lohmueller, C. Heske, E. Umbach, W. Riedl, S. Zweigart, F. Karg, Flat conduction-band alignment at the $\mathrm{CdS} / \mathrm{CuInSe} 2$ thin-film solar-cell heterojunction, Applied Physics Letters 79 (27) (2001) 4482. doi:10.1063/1.1428408.

[30] S. Chen, A. Walsh, X.-G. Gong, S.-H. Wei, Classification of Lattice Defects in the Kesterite $\mathrm{Cu}_{2} \mathrm{ZnSnS}_{4}$ and $\mathrm{Cu}_{2} \mathrm{ZnSnSe}_{4}$ Earth-Abundant Solar Cell Absorbers, Advanced Materials 25 (11) (2013) 1522-1539. doi:10.1002/adma.201203146.

[31] R. Scheer, H.-W. Schock, Chalcogenide Photovoltaics, Wiley-VCH Verlag, Weinheim, Germany, 2011. doi:10.1002/9783527633708.

[32] A. Crovetto, M. K. Huss-Hansen, O. Hansen, How the relative permittivity of solar cell materials influences solar cell performance, Solar Energy 149 (2017) 145-150. doi:10.1016/j.solener.2017.04.018.

33] Y. F. Tay, H. Kaneko, S. Y. Chiam, S. Lie, Q. Zheng, B. Wu S. S. Hadke, Z. Su, P. S. Bassi, D. Bishop, T. C. Sum, T. Minegishi, J. Barber, K. Domen, L. H. Wong, SolutionProcessed Cd-Substituted CZTS Photocathode for Efficient Solar Hydrogen Evolution from Neutral Water, Joule 2 (3) (2018) 537-548. doi:10.1016/j.joule.2018.01.012.

[34] W. Shockley, H. J. Queisser, Detailed Balance Limit of Efficiency of p-n Junction Solar Cells, Journal of Applied Physics 32 (3) (1961) 510. doi:10.1063/1.1736034.

[35] S. Tajima, R. Asahi, D. Isheim, D. N. Seidman, T. Itoh, M. Hasegawa, K. Ohishi, Atom-probe tomographic study of interfaces of $\mathrm{Cu}_{2} 2 \mathrm{ZnSnS}_{4}$ photovoltaic cells, Applied Physics Letters 105 (9) (2014) 093901. doi:10.1063/1.4894858. 